\title{
State Machine
}

National Cancer Institute

\section{Source}

National Cancer Institute. State Machine. NCI Thesaurus. Code C81926.

A model of computation consisting of a set of states, a set of input events, a set of output events, and a state transition function. 